\title{
Protocol for a Meta-narrative Review on Research Paradigms Addressing the Urban Built Environment and Human Health
}

Jinhee Kim ( $\nabla_{\text {jinhee.kim@unsw.edu.au ) }}$

University of New South Wales https://orcid.org/0000-0002-6439-792X

Ben Harris-Roxas

University of New South Wales

Evelyne de Leeuw

University of New South Wales

David Lilley

University of New South Wales

Alana Crimeen

University of New South Wales

Peter Sainsbury

The University of Notre Dame Australia School of Medicine Sydney Campus

\section{Protocol}

Keywords: paradigms, urban health, healthy cities, healthy urban planning, health social movements, medical-industrial complex, meta-narrative review

Posted Date: December 8th, 2020

DOI: https://doi.org/10.21203/rs.3.rs-121888/v1

License: (c) (1) This work is licensed under a Creative Commons Attribution 4.0 International License. Read Full License

Version of Record: A version of this preprint was published at Systematic Reviews on December 1st, 2021. See the published version at https://doi.org/10.1186/s13643-021-01848-6. 


\section{Abstract}

\section{Background}

Urban health is a field of research and practice that has attracted the interest of various disciplines. While it is encouraged for diverse disciplines to contribute to a multidisciplinary field of study such as urban health, this often results in tensions, conflicts or competition between the different traditions that stem from different epistemological backgrounds.

This meta-narrative review aims to identify and describe the multiple paradigms and articulate the underlying epistemological, ontological, methodological and aetiological differences in their approaches. Articulating the paradigms not only contributes to the advancement of research, but also provides a framework for understanding the different policy beliefs and ideas policy actors hold and apply in the policy process.

\section{Methods}

We apply the meta-narrative method to systematic literature review which includes the following six iterative phases. The planning phase includes the finalisation of the review protocol and assembly of review team. The search phase includes a comprehensive literature search in key databases and a double-sided systematic snowballing method. Bibliometric analyses of this literature will be used to triangulate the mapping of the paradigms. The mapping phase includes identifying the dominant paradigms and landmark publications through agreement with the review team. In the appraisal phase, the literature will be assessed by their respective quality standards, followed by data extraction to identify the individual narratives in the conceptual, theoretical, methodological and instrumental dimensions of each paradigm. The synthesis phase will review the data to compare and contrast and identify the overarching meta-narratives. The recommendation phase will include dissemination of the findings from the review.

\section{Discussion}

The meta-narrative review will reveal the how the different paradigms conceptualise, frame and prioritise urban health issues, their preferred methodologies to study the phenomenon, and the nature of the solutions to improve human health. This review will assist researchers and practitioners in understanding and interpreting evidence produced by other traditions that study urban health. Through this, urban health researchers and practitioners will be able to seek coherence in understanding, explaining and exploring the urban health phenomenon.

Systematic review registration

PROSPERO CRD42020192992

\section{Background}


The research and practice of urban health involves contributions from multiple disciplines, sectors and trades that represent different aspects of urban health phenomena. Each discipline, sector or trade has a role in the complex interplay between the urban built environment and its relationship to health and health equity impacts. While it is encouraged for actors from diverse backgrounds to contribute to a multidisciplinary field of study such as urban health, collaboration efforts sometimes result in tensions, conflicts and competition. This is because disciplines and sectors have historically evolved in silos and branched out as specialisations that have developed different standards of 'normal science', or practices or empirical approaches members of a certain discipline or sector take for granted (1). The different standards are strongly rooted in the epistemological, ontological, methodological and aetiological definitions of urban health of the diverse disciplines that often act as barriers to meaningful interdisciplinary or intersectoral collaboration (2-4).

These positions are defined as paradigms, or particular frameworks that researchers apply to understand the complexity of the real world. In a given paradigm, a distinct set of concepts and practices provides a common framework for addressing problems and solutions (1). The set of concepts of a paradigm defines what is regarded as important issues that require attention in urban health and which study designs and methods are the best way to produce the required knowledge. Therefore, a group of researchers and practitioners that belong to a paradigm share a set of rules and standards that are selfevident but are incommensurable with other paradigms. That is, empirical findings that were produced using one set of concepts, theories, methods and instruments may not only be inapplicable to issues seen important in other paradigms but unacceptable to the followers of a different paradigmatic lens.

In our preliminary review of the literature, we identify four prominent paradigms in the study and policies that address the impact of the urban built environment on human health. The approaches of the 'medicalindustrial city paradigm focuses on the development of healthcare facilities as a key urban planning project in the city or the application of technology to the urban infrastructure to monitor or change disease, risk factors and behaviour of individuals. The 'urban health science' paradigm applies epidemiological and complex systems analyses to urban health issues. Here, conclusive empirical data and analyses that confirm the causal relationships between the urban built environment and human health outcomes is prioritised and is used as evidence to develop effective interventions and policies. The 'healthy built environment' paradigm originates from the urban planning discipline and advocates for the integration of health in the practice of spatial planning of cities. The 'health social movement' paradigm seeks to integrate health considerations into all aspects of urban governance, with an emphasis on operationalising values such as health equity and empowerment. This typology has been developed from an earlier version in which we had identified the latter three paradigms (5)

Other studies have similarly confirmed the co-existence of multiple approaches in this field. From the articles retrieved via a search in Pubmed/MEDLINE of the MeSH term "urban health", Jia et al. (6) identified four distinct categories of urban health research as physical environment, health impacts, social environments and interventions. Forsyth (7) identified three conceptually distinct categories of healthy places approaches, i.e. basic healthy places (developing a physical and/or institutional structure 
supportive of health), population-based lenses (focus on population groups with health vulnerabilities and wide relevance), and technology-focused places (harnessing innovative technology to create a healthy economy and/or assist in health monitoring and promotion).

In contrast to the distinctions in subject matter made by the above authors, our emphasis is on identifying and describing the multiple paradigms and articulating the underlying epistemological, ontological, methodological and aetiological differences in their approaches. This is particularly important for the field of urban health because maximising health gains cannot be effectively achieved by merely working within the common intersecting areas of specific disciplines and sectors. Urban health is a multidisciplinary field of research and practice that requires more attention and understanding in the non-overlapping areas. This meta-narrative review attempts to study how the topic of the urban built environment and human health has been differently conceptualised and researched by different traditions.

Articulating the paradigms not only contributes to the advancement of research, but also provides a framework for understanding the different policy beliefs and ideas policy actors hold and apply in the policy process. Ideas are organized into policy paradigms which sometimes have the power to induce changes to the institutional routines (8). Moreover, understanding the key dimensions of the different urban health paradigms can help to prevent answering the research question correctly but forming a wrong interpretation or response (9).

\section{Purpose and research questions}

For the purpose of this study, we limit the scope of the urban health phenomenon to approaches in research and practice that address the issues concerned with the impact of the urban built environment on human health in cities or urban areas. In this meta-narrative review, we aim to identify the different contemporary urban health paradigms and articulate the characteristics in their conceptualisation, theoretical framework, methodological approaches and instrumental solutions to urban health issues. The units of analysis are the different urban health paradigms and publications are the data source to examine their characteristics.

The primary research question of this review is: What are the dominant paradigms in research and practice on the issue of the urban physical environment and its impact on human health? Secondary research questions involve identifying the characteristics typical of each paradigm, such as (1) What are considered the important objects of study in each paradigm? (2) How are urban built environments and their impact on human health conceptualised? (3) Which methodological approaches are preferred? (4) What is the nature of the policy solutions?

\section{Methods/design}

To understand the heterogeneity of research traditions that study urban health, we apply the metanarrative approach to systematic literature review. A meta-narrative review is a type of systematic 
literature review that is designed for topic areas that are researched by diverse research traditions, with different conceptualisations and methodologies (10-13). Through an explicit, rigorous and transparent process, meta-narrative reviews identify, articulate, synthesise and interpret a diverse body of literature in a topic area $(11,14,15)$. In this review, we will systematically collect and analyse the literature that addresses the issues concerning the impact of urban built environments on human health and seek to make sense of the complex and contested knowledge in this topic area.

The protocol for this review was developed in accordance with the RAMESES (Realist And MEta-narrative Evidence Syntheses: Evolving Standards) publication standards (15) and quality criteria suggested in the associated meta-narrative review training materials (14). Because meta-narrative reviews are different from traditional systematic literature reviews that they are designed to reflect the heterogeneity of the research methodologies, the protocol is not fully compatible with the Preferred Reporting Items for Systematic Review and Meta-Analysis Protocol (PRISMA-P) guidelines. Nevertheless, we have populated the PRISMA-P checklist as it still provides critical value to the systematic review process [Additional file]. The protocol is registered with PROSPERO [CRD42020192992].

The six guiding principles of the meta-narrative review (15) are integrated into the review process as articulated below (Table 1). 
Table 1

Meta-narrative review principles and applications

\begin{tabular}{|c|c|c|}
\hline Principle & Definition & Application in this review \\
\hline Pragmatism & $\begin{array}{l}\text { The review should } \\
\text { address what will } \\
\text { be most useful to } \\
\text { the intended } \\
\text { audience }\end{array}$ & $\begin{array}{l}\text { The objective of this review is to understand the main } \\
\text { paradigms in urban health. In a transdisciplinary field of } \\
\text { research and practice, articulating the non-overlapping } \\
\text { characteristics of different paradigms is critical to attain } \\
\text { coherence and collaboration across disciplines, sectors and } \\
\text { paradigms. }\end{array}$ \\
\hline Pluralism & $\begin{array}{l}\text { The topic should be } \\
\text { illuminated from } \\
\text { multiple angles and } \\
\text { perspectives }\end{array}$ & $\begin{array}{l}\text { We explore the current knowledge base in various disciplines, } \\
\text { including public health, urban planning, local/city governance } \\
\text { and urban studies. A list of relevant disciplines and journals } \\
\text { will be drafted to utilize for the hand selecting of literature to } \\
\text { avoid any exclusion of the disciplines. }\end{array}$ \\
\hline Historicity & $\begin{array}{l}\text { The deepest } \\
\text { understanding of a } \\
\text { topic comes from } \\
\text { studying its } \\
\text { evolution over time }\end{array}$ & $\begin{array}{l}\text { The genealogy and clusters of the literature will be analysed } \\
\text { using bibliometric methods. Landmark documents will be } \\
\text { recorded and traced to study the evolution of the paradigms. }\end{array}$ \\
\hline Contestation & $\begin{array}{l}\text { Conflicting data } \\
\text { from different } \\
\text { research traditions } \\
\text { should be } \\
\text { examined to } \\
\text { generate higher- } \\
\text { order insights }\end{array}$ & $\begin{array}{l}\text { Differences between the conceptualisation of urban health, } \\
\text { causal pathways, methodological approaches and policy } \\
\text { solutions will be highlighted. Details on the application of this } \\
\text { principle will be explored further in the data extraction, } \\
\text { analysis and synthesis phases. }\end{array}$ \\
\hline Reflexivity & $\begin{array}{l}\text { Reviewers should } \\
\text { continually reflect } \\
\text { on the emerging } \\
\text { findings }\end{array}$ & $\begin{array}{l}\text { The protocol will be updated to reflect the changes to the } \\
\text { process as findings emerge. Any changes made to the review } \\
\text { that were initially planned will be described and justified in the } \\
\text { final report. }\end{array}$ \\
\hline Peer review & $\begin{array}{l}\text { Emerging findings } \\
\text { should be } \\
\text { presented and } \\
\text { discussed with an } \\
\text { external audience }\end{array}$ & $\begin{array}{l}\text { The emerging findings will be communicated with peers via } \\
\text { individual consultations with experts and presentations at } \\
\text { conferences and meetings. A website will be developed as a } \\
\text { platform for the wider community to engage in the process as } \\
\text { well as for dissemination of the findings. }\end{array}$ \\
\hline
\end{tabular}

The methods section of this protocol is presented according to the six phases of a meta-narrative review recommended by the RAMESES publication guideline.

\section{Planning phase}

A review team consisting of the authors of this protocol was assembled, who developed the research questions and drafted the protocol. The review team provided feedback on the draft protocol. Knowledge users, defined as the broader network of researchers and practitioners in the field of urban health, will be approached to serve as external expert panel members for the review and will be consulted if additional assistance is needed. 


\section{Search phase}

\section{Eligibility criteria}

Publications to be included in the review will be limited by language (English) and publication types (journal articles, reviews, books, book chapters, editorial and opinion pieces and reports). All study designs, including empirical and non-empirical studies, and all publication years will be considered for inclusion. The topic of the paper must explicitly focus on urban human-constructed physical environments and human health at the city or urban scale and must address one or more of the conceptual, theoretical, methodological or instrumental dimensions on this topic.

\section{Search strategy}

The main objective of the search is to collect a comprehensive list of the literature on the topic area to capture the diversity of the urban health research traditions and paradigms. The publications will serve as primary data source to analyse the meta-narratives of each paradigm. The balance between comprehensiveness and precision of the search is resolved by applying the concept of saturation as the criterion, a concept borrowed from qualitative research methods. Saturation is achieved when no new information is generated by collecting additional data. Because this review is a "knowledge-building and theory-generating' type of systematic review, there is no intrinsic value in continuing the search unless there is additional theoretical contribution (16). This is in contrast to those reviews which study the aggregation or summation of concepts, where 'the more the better' approach is preferred. The attainment of saturation will be determined at the appraisal or synthesis phase when the review team observes a conceptual saturation of the findings from the identified literature, and decide that the addition of unidentified studies will only contribute to marginal changes to the findings. (17-19).

The search will take three main strategies - (a) a double-sided snowballing search, (b) a search in electronic databases using search terms, and (c) an additional hand search. The double-sided snowballing search will include a forward search of all papers that cite the landmark work identified in the mapping phase, and a backward search that collects the literature included in the reference list of these papers (20). A search using keyword search terms will be conducted in relevant multidisciplinary scientific databases (e.g. ProQuest, Web of Science (Science and Social Sciences Citation Indices), Scopus) and topic-specific databases (e.g. Urban Studies Abstracts - EBSCO, Medline - Ovid, Embase Ovid). The search terms will include those related to city ("city" OR "cities" OR "urban" OR "local" OR "municipal") combined with concepts on the built environment ("built environment" OR "physical environment" OR "infrastructure" OR "planning" OR "design") and search terms related to health and health equity. An additional hand search of key journals and publications by key organisations will be conducted to maximise comprehensiveness.

\section{Selection process}


The publications identified from these three strategies will be compiled in EndNote and exported to Covidence to be screened for inclusion in the review. Two reviewers will screen the title and abstract of each publication to decide inclusion in the review. Any disagreement will be resolved by consensus. Details of the inclusion criteria will be added and refined as the reviewers proceed with the screening. It is also expected that the search strategy will be iteratively revised by the paradigms identified in the mapping phase.

\section{Mapping phase}

\section{Mapping urban health paradigms and defining parameters}

As mentioned earlier, we start with four paradigms on urban health - the medical-industrial city, urban health science, healthy built environments and health social movement - as the initial paradigms for this review (Table 2). These four paradigms explicitly address the relationship between the urban built environment and human health, and each has a set of conceptual, theoretical, methodological and instrumental dimensions. Findings from the search and discussions with the wider expert community may introduce additional paradigms to the review. For example, there may be additional paradigms in the fields of environmental health, spatial justice, or civil engineering which were not covered in our initial mapping. Alternatively, the initial paradigms may merge or subdivide as more information is added from the review process.

Table 2

Four initial urban health paradigms

\begin{tabular}{|lllll|}
\hline & $\begin{array}{l}\text { Medical- } \\
\text { industrial city }\end{array}$ & Urban health science & $\begin{array}{l}\text { Healthy built } \\
\text { environments }\end{array}$ & $\begin{array}{l}\text { Health social } \\
\text { movements }\end{array}$ \\
\hline $\begin{array}{l}\text { Main } \\
\text { concept }\end{array}$ & $\begin{array}{l}\text { The healthcare } \\
\text { industry is a } \\
\text { main driver for } \\
\text { urban growth } \\
\text { and } \\
\text { development. }\end{array}$ & $\begin{array}{l}\text { Production of evidence } \\
\text { that reveal and confirm } \\
\text { causal relationships } \\
\text { between the built } \\
\text { environment and health. }\end{array}$ & $\begin{array}{l}\text { Influencing the } \\
\text { planning system } \\
\text { to integrate } \\
\text { health to urban } \\
\text { planning } \\
\text { decisions. }\end{array}$ & $\begin{array}{l}\text { Empowering the } \\
\text { community and } \\
\text { value-based } \\
\text { decision-making } \\
\text { for healthy cities. }\end{array}$ \\
\hline $\begin{array}{l}\text { Related } \\
\text { disciplines } \\
\text { and fields }\end{array}$ & $\begin{array}{l}\text { Business, urban } \\
\text { planning, } \\
\text { development, } \\
\text { economics }\end{array}$ & $\begin{array}{l}\text { Public health, } \\
\text { epidemiology, population } \\
\text { health, implementation } \\
\text { science }\end{array}$ & $\begin{array}{l}\text { Urban planning, } \\
\text { urban design, } \\
\text { land-use } \\
\text { planning }\end{array}$ & $\begin{array}{l}\text { Health promotion, } \\
\text { community } \\
\text { development, } \\
\text { social justice, } \\
\text { sociology }\end{array}$ \\
\hline
\end{tabular}

In the mapping phase, we will develop a set of parameters for each paradigm. For example, we will define the characteristics of each paradigm based on their definitions of urban health, the theoretical frameworks that explain the relationship between urban physical environments and health, the methodologies to research (e.g. what is counted as knowledge, evidence, etc.) and the solutions such as the policies, practice and solutions to improve urban health. In particular, we will include the concepts of health equity and the consideration of power in decision making in reviewing the parameters. These 
parameters will be used as a guide to search for data on the dimensions of the multiple paradigms in each publication. We will apply the set of parameters to assign each piece of an included publication to its corresponding paradigm(s). To ensure high inter-coder reliability, the review team will first code a small sample of the publications and any conflicts or disagreement will be examined and discussed. Any disagreement will be resolved by consensus within the review team.

\section{Bibliometric analysis to visualise network and clusters}

A supplementary bibliometric analysis using the dataset of the final set of articles will be conducted to map the genealogy of citations and the author network analysis (21). The findings will visualise clusters of researchers and relationships between publications. These visualisation data will provide information to triangulate the different paradigms and research traditions.

\section{Identifying landmark works}

The mapping phase includes identifying landmark works that formed the foundation for the paradigms and are recognised by scholars in the field as highly influential in shaping subsequent research and practice (11). They can be conceptual papers or reports, or empirical studies that formed a model for future work in the paradigm. We will triangulate this with the citation metrics data and the findings from the bibliometric network analysis.

The following inclusion criteria will be applied to identify the landmark sources (11)

1. Is the paper part of a recognised research paradigm, that is, does it draw critically and comprehensively upon an existing body of scientific knowledge and attempt to further that body of knowledge?

2. Does the paper make an original and scholarly contribution to research into the topic area?

3. Has the paper subsequently been cited as a landmark contribution (conceptual, theoretical, methodological or instrumental) by competent research in that tradition?

4. Is the paper an exemplar of a recognised research paradigm and its parameters?

The review team will independently score and nominate landmark sources according to the above criteria. Discussions will be held with external experts to attain consensus.

\section{Appraisal phase}

In the appraisal phase, we will extract data from selected publications by coding the conceptual, theoretical, methodological and instrumental concepts. The main outputs from this phase include a codebook with the descriptions of the codes, an NVivo project with coded data of the included literature, development of the quality assessment criteria for each paradigm, and the quality assessment of the literature.

\section{Data extraction}


First, a code system (or a data extraction template) will be developed based on the parameters of the paradigms developed in the mapping phase. The code system and data extraction template will include:

- Bibliometric meta data (e.g. author, publication year, title, type of publication, etc.)

- Research questions and how they were framed, and conceptual and theoretical issues;

- Preferred methodologies, study designs and quality criteria;

- Key actors (e.g. leading scientists or commentators) and events (e.g. conferences) in the unfolding of the tradition;

- Landmark empirical or theoretical studies;

- Significant findings and how they shaped subsequent work;

- Key debates and areas of dispute within the tradition, including links with or breaches from other traditions.

- Characteristics in cross-disciplinary approaches (interdisciplinary, multidisciplinary or transdisciplinary).

Details of the coding system will be determined by the review team. Whether data extraction will be based on the abstract or the full-text will be determined at a later time, after the review team learns more about the body of literature. We will use the NVivo qualitative data analysis software to efficiently organise the data. Using NVivo software for qualitative coding will also allow us to refer back to the original data and transparently track the collaborative process. The reviewers will independently extract data and the coded data will be examined to ensure inter-coder reliability. All data will be stored in the approved research data storage system provided by the lead author's institution and handled in accordance with the institution's data management standards and guidelines.

\section{Quality and risk assessment}

Each paradigm will inherently endorse a different set of standards for assessing the quality and risk of bias of studies. Criteria to assess the quality and risk of bias will be taken from the paradigms included in the review, particularly from the landmark papers that have been accepted by the paradigm as authoritative. The publications, now classified to one or more paradigm, will be assessed against the corresponding quality criteria.

\section{Synthesis phase}

\section{Building the meta-narratives of each paradigm}

Synthesis involves comparing and contrasting the meta-narratives among the different paradigms to identify and compare how they have conceptualised the topic, how they have theorised it, and the methodological approaches and study designs used. After coding the data from the primary studies within each paradigm, we will construct a meta-narrative for each paradigm, and then compare and contrast the paradigms. 


\section{Comparing meta-narratives across paradigms}

Synthesis across paradigms may occur at a high level of abstraction and may involve one or more of the following:

- Paradigm bridging (seeking commonalities in conceptual and theoretical assumptions)

- Paradigm bracketing (highlighting differences in these assumptions)

- Interplay (exploring tensions)

- Meta-theorizing (exploring patterns that span conflicting understandings)

The key questions we ask in this phase include: (1) What is the range of questions the paradigms address across the four dimensions? (2) What are the commonalities and conflicts of research findings across the paradigms and how can the discrepancies be explained? (3) What are the overall key findings and implications? (4) What are the main gaps and where should future research be directed?

\section{Recommendation phase}

The final phase of the review includes drafting the final report with key messages and recommendations for practice, policy and further research. The final report will be developed through reflection and discussions with the review team and feedback from the wider urban health epistemological community. Reporting will be in compliance with the RAMESES publication standards (15). Any changes made to the protocol will be documented in the final review report.

A dissemination strategy will be developed to further communicate with the broader knowledge user community. Components of the review (e.g. the protocol, methodology, meta-narrative review findings) will be reported through traditional academic communication channels such as academic journal publications and conference presentations. Presenting the findings of the review over the various stages will provide a form of triangulation to ensure the validity of the review and address some of the issues occurring from meta-biases such as publication bias or selective reporting. The team will further identify a broad range of potential knowledge users and stakeholders and develop innovative strategies to effectively communicate with them.

\section{Discussion}

To our knowledge there has not been a comprehensive search of the literature that identifies the different approaches to urban health issues and their solutions. By systematically reviewing the literature we will be able to identify the different paradigms within which researchers and policy actors address urban health issues and develop a comprehensive map of the field. Paradigms are not only relevant in research and science, but also have a fundamental role in forming the policy ideas and beliefs of the actors involved in the policy process $(8,22-25)$. The findings from this review will contribute to highlighting conflicting evidence between the paradigms and finding gaps in the approaches. Detailed articulation of 
the paradigms will facilitate communication and knowledge transfer between previously incommensurable paradigms.

In the development of this protocol, we adhered to the RAMESES publication guideline and its quality standards (15). However, in some cases, we found that the meta-narrative approach that we apply in our review is not completely compatible with the required reporting templates for systematic reviews such as PROSPERO, the International Prospective Register of Systematic Reviews, or the Preferred Reporting Items for Systematic Review and Meta-Analysis Protocol (PRISMA-P). For example, because the units of analysis of a meta-narrative review are paradigms, it is not appropriate to identify the PICO elements Participants, Intervention, Comparison, Outcomes - which are elements typical of systematic reviews that study the effectiveness of interventions often in clinical settings. Also, because the execution of metanarrative reviews is iterative by nature, that is, findings from a subsequent phase will often provide information about or for a previous phase, these processes is often challenging to describe in the templates that were designed for a more linear process. Similarly, as we plan to take a reflective and interpretive approach by presenting our preliminary findings to other colleagues within the broader urban health research community for their feedback throughout the different stages of the review, these iterative and interpretive characteristics were not effectively captured in the PROSPERO or PRISMA-P reporting guidelines. Lastly, because meta-narrative reviews include studies across different paradigms and study designs, the criteria applied to assess the studies must inevitably be selected according to the standards of each paradigm and research tradition.

In summary, this meta-narrative review will reveal how the different paradigms conceptualise, frame and prioritise urban health issues, their preferred methodologies to study the phenomenon, and the nature of the solutions to improve human health. The findings from the review will assist researchers and practitioners in understanding and interpreting evidence produced by other paradigms that study urban health. Through this, urban health researchers and practitioners will be able to seek coherence in understanding, explaining and exploring the urban health phenomenon.

\section{List Of Abbreviations}

PRISMA-P Preferred Reporting Items for Systematic Review and Meta-Analysis Protocol

RAMESES Realist And MEta-narrative Evidence Syntheses: Evolving Standards

\section{Declarations}

\section{Ethics approval and consent to participate}

Not applicable

\section{Consent for publication}




\section{Availability of data and materials}

Not applicable

\section{Competing interests}

The authors declare that they have no competing interests.

\section{Funding}

This work was supported by the UNSW Scientia Scheme. The funder had no involvement in the conduct of the research nor in the preparation of the manuscript.

\section{Authors' contributions}

JK lead the conception and design of the systematic review with the intellectual and technical support of BH-R, EdL and PS. JK drafted the protocol manuscript, and all authors (BHR, EdL, DL, AC and PS) provided comments and contributed to the revisions. All authors read and approved the final version before submission.

\section{References}

1. Kuhn T. The structure of scientific revolutions. Chicago: University of Chicago Press; 1962.

2. Lawrence R, Gatzweiler F. Wanted: a transdisciplinary knowledge domain for urban health. J Urban Health. 2017;94:592-6.

3. O'Campo P, Kirst M, Schaefer-McDaniel N, Hwang S. Introducing a transdisciplinary approach to applied urban health research. In: Kirst M, Schaefer-McDaniel N, Hwang S, O'Campo P, editors. Converging disciplines: a transdisciplinary research approach to urban health problems. New York, NY: Springer; 2011. p. 3-11.

4. Ramadier T. Transdisciplinarity and its challenges: the case of urban studies. Futures. 2004;36(4):423-39.

5. Kim J, de Leeuw E, Harris-Roxas B, Sainsbury P. The three paradigms on urban health. Eur J Public Health. 2020 Sep 1;30(Supplement_5):v586-7.

6. Jia X, Dai T, Guo X. Comprehensive exploration of urban health by bibliometric analysis: 35 years and 11,299 articles. Scientometrics. 2014 Jun 1;99(3):881-94. 
7. Forsyth A. What is a healthy place? Models for cities and neighbourhoods. J Urban Des. 2020;25(2):186-202.

8. Hall PA. Policy paradigms, social learning, and the state: the case of economic policymaking in britain. Comp Polit. 1993;25(3):275-96.

9. de Leeuw E. Do healthy cities work? A logic of method for assessing impact and outcome of healthy cities. J Urban Health Bull N Y Acad Med. 2012;89(2):217-31.

10. Chughtai S, Blanchet K. Systems thinking in public health: a bibliographic contribution to a metanarrative review. Health Policy Plan. 2017;32(4):585-94.

11. Greenhalgh T, Robert G, Macfarlane F, Bate P, Kyriakidou O, Peacock R. Storylines of research in diffusion of innovation: a meta-narrative approach to systematic review. Soc Sci Med. 2005;61(2):417-30.

12. Jones A, Hannigan B, Coffey M, Simpson A. Traditions of research in community mental health care planning and care coordination: A systematic meta-narrative review of the literature. PLOS ONE. 2018;13(6):e0198427.

13. Van Belle S, Mayhew SH. What can we learn on public accountability from non-health disciplines: a meta-narrative review. BMJ Open. 2016 Jul;6(7):e010425.

14. Greenhalgh T, Wong G. Training materials for meta-narrative reviews [Internet]. 2013. Available from: http://www.ramesesproject.org/media/Meta_narrative_reviews_training_materials.pdf

15. Wong G, Greenhalgh T, Westhorp G, Buckingham J, Pawson R. RAMESES publication standards: meta-narrative reviews. BMC Med. 2013;11(1):20.

16. Finfgeld-Connett D, Johnson ED. Literature search strategies for conducting knowledge-building and theory-generating qualitative systematic reviews: discussion paper. J Adv Nurs. 2013;69(1):194-204.

17. Maxwell JA. Qualitative research design: an interactive approach. SAGE Publications; 2012.

18. Denzin N, Lincoln Y. The SAGE handbook of qualitative research. 5th ed. SAGE; 2017.

19. Flick U. Managing quality in qualitative research - SAGE research methods. London: SAGE Publications, Inc.; 2007.

20. Greenhalgh T, Peacock R. Effectiveness and efficiency of search methods in systematic reviews of complex evidence: audit of primary sources. BMJ. 2005;331(7524):1064-5.

21. van Eck NJ, Waltman L. Citation-based clustering of publications using CitNetExplorer and VOSviewer. Scientometrics. 2017;111(2):1053-70.

22. Barnett C, Parnell S. Ideas, implementation and indicators: epistemologies of the post-2015 urban agenda. Environ Urban. 2016;28(1):87-98.

23. Baumgartner FR. Ideas, paradigms and confusions. J Eur Public Policy. 2014;21(3):475-80.

24. Daigneault P-M. Reassessing the concept of policy paradigm: aligning ontology and methodology in policy studies. J Eur Public Policy. 2014;21(3):453-69.

25. Wilder M. What is a policy paradigm? Overcoming epistemological hurdles in cross-disciplinary conceptual adaptation. In: Hogan J, Howlett M, editors. Policy Paradigms in Theory and Practice: 
Discourses, Ideas and Anomalies in Public Policy Dynamics. London: Palgrave Macmillan UK; 2015. p. $19-42$.

\section{Supplementary Files}

This is a list of supplementary files associated with this preprint. Click to download.

- PRISMAPchecklist.docx 